BRIEF COMMUNICATION

\title{
The interaction of lipids and inflammatory markers predict negative symptom severity in patients with schizophrenia
}

\author{
David R. Goldsmith $\mathbb{D}^{1 凶}$, Nicholas Massa (D) $^{2}$, Brian J. Miller ${ }^{3}$, Andrew H. Miller $\mathbb{D}^{1}$ and Erica Duncan ${ }^{1,2}$
}

Finding biological predictors and novel mechanisms underlying negative symptoms of schizophrenia is of significant importance given the lack of effective treatments. Increasing data support a role for metabolic dysfunction and inflammation in reward processing deficits in psychiatric illness. Herein, we found an interaction between lipids and inflammation as a predictor of worse negative symptom severity in individuals with schizophrenia. Future studies may seek to further elucidate this relationship and thereby reveal novel treatment targets for negative symptoms.

npj Schizophrenia (2021)7:50; https://doi.org/10.1038/s41537-021-00179-8

There is increased focus on the interaction of inflammatory and metabolic pathways in psychiatric disorders ${ }^{1-4}$. Individuals with schizophrenia consistently show disruptions of several metabolic parameters, including lipid and glucose homeostasis, which may be associated with underlying genetic risk for the disorder ${ }^{5,6}$. Similarly, many individuals with schizophrenia have increased inflammatory markers ${ }^{7}$, which have previously been associated with negative symptom severity ${ }^{8}$.

Both systemic inflammation and metabolic disturbances have effects on the brain ${ }^{9,10}$. Our group has previously shown that peripheral biomarkers and gene transcripts related to glucose and lipid metabolism predicted response to the antiinflammatory effects of infliximab in patients with depression ${ }^{4,11}$. We have also recently demonstrated that markers of altered glucose metabolism were associated with decreased functional connectivity in reward circuitry, an effect that was only seen in individuals with increased inflammation in patients with depression ${ }^{2}$.

These relationships have not been investigated relative to negative symptoms of schizophrenia, which are also marked by alterations in reward processing ${ }^{12}$. Accordingly, we examined negative symptoms and non-fasting levels of plasma lipid markers as well as plasma concentrations of two of the most commonly altered cytokines in patients with schizophrenia: tumor necrosis factor (TNF) and interleukin-6 (IL-6), which have both been shown to be associated with negative symptoms ${ }^{13,14}$. We also repeated the analyses in a second independent dataset in order to replicate these findings.

Sociodemographic and clinical variables as well as immune and metabolic marker concentrations for the primary and replication sample are shown in Table 1.

Of the metabolic markers, only total cholesterol $(r=0.387, p=$ $0.005)$ and LDL $(r=0.356, p=0.009)$ were significantly correlated with the PANSS negative subscale score (Fig. 1). No metabolic markers were correlated with total PANSS score or PANSS positive or general scores. As such, only total cholesterol and $\mathrm{LDL}$, as well as PANSS negative subscale score, were used in subsequent analyses. Cholesterol and LDL had evidence of increased kurtosis/skewness, and as such both values were logtransformed and mean-centered for use in all further analyses. Neither TNF, IL-6, or their combination were significantly correlated with total, positive, negative, and general PANSS scores or individual subscale scores.

The sample was split into a high and low inflammation group based on a median split of the $z$-scored combination of TNF and IL-6. There was no relationship between PANSS negative symptoms and either cholesterol $(r=0.134, p=0.514)$ or LDL $(r=0.204, p=0.317)$ in the low inflammation group. In the high inflammation group, there was a significant relationship between PANSS negative symptoms and both cholesterol $(r=0.599, p=$ $0.001)$ and LDL ( $r=0.582, p=0.002)$ (Fig. 1).

In order to test the interaction effect between inflammatory and metabolic markers, we conducted linear regression models including cytokine group, metabolic marker group, and their interaction in addition to relevant covariates. There was a significant interaction between the TNF group and the LDL group for negative symptoms $(p<0.05)$, but not for the other markers. The TNF + IL- 6 group $\times$ LDL group significantly predicted negative symptoms $(\beta=1.533, p=0.016)$ as well as individual negative symptom items including blunted affect $(r=0.293, p=0.035)$ and emotional withdrawal $(r=0.346, p=0.012)$.

In the replication sample, the TNF + IL- 6 group $\times$ cholesterol group interaction correlated with emotional withdrawal $(r=0.280$, $p=0.030)$ as did the TNF + IL- 6 group $\times$ LDL group interaction $(r=0.280, p=0.030)$, controlling for age, sex, race, smoking, and BMI (see Supplementary Materials for more detail).

This study demonstrated interactive relationships between lipid metabolism and inflammatory markers predicting negative symptom severity in a sample of patients with schizophrenia. We were also able to replicate findings in a second, independent dataset. Though we have previously shown relationships between increased inflammation and negative symptoms of schizophrenia, this is the first study to support a role for a relationship between negative symptoms and lipid metabolism in patients with schizophrenia that is dependent on inflammation. This is consistent with recent evidence that high triglycerides and high inflammation together predicted negative symptom severity and worse outcomes at 1-year follow-up in first episode psychosis ${ }^{3}$. Obesity and metabolic dysfunction drive systemic inflammation via the activation of macrophages in adipose tissue ${ }^{15}$. Inflammatory cytokines released by macrophages play a critical role in the development of hyperlipidemia

\footnotetext{
${ }^{1}$ Emory University School of Medicine, Department of Psychiatry and Behavioral Sciences, Atlanta, GA, USA. ${ }^{2}$ Atlanta Veterans Affairs Health Care System, Decatur, GA, USA.
}

${ }^{3}$ Augusta University, Department of Psychiatry and Health Behavior, Augusta, GA, USA. ${ }^{凶}$ email: drgolds@emory.edu 
and insulin resistance, which in turn, leads to metabolic disturbances that further promote cytokine production from adipose tissue, thus contributing to chronic inflammation ${ }^{16}$. Though this study was limited by small sample sizes, future work should seek to further investigate the bidirectional impact of inflammation and metabolic dysfunction as a driver of negative

Table 1. Sociodemographic and clinical variables as well as immune and metabolic marker concentrations in the primary sample $(n=52$ patients) and the replication sample $(n=65)$.

\begin{tabular}{lcl}
\hline & Primary sample & Replication sample \\
\hline Age & $51.3(9.2)$ & $41.69(12.4)$ \\
Sex & $49 / 52$ male $(94.2 \%)$ & $34 / 65$ male $(52.3 \%)$ \\
Race & $39 / 52$ black $(75 \%)$ & $46 / 65$ black $(70.8 \%)$ \\
Smoking & $26 / 52$ smoker $(50 \%)$ & 8 cigarettes smoked daily (9.5) \\
BMI & $31.4(6.8)$ & $32.2(7.7)$ \\
PANSS total score & $60.6(14.5)$ & $70.9(16.7)$ \\
PANSS positive subscale score & $15.8(4.8)$ & $18.8(6.4)$ \\
PANSS negative subscale score & $16.6(6.0)$ & $16.1(5.8)$ \\
PANSS general subscale score & $28.2(7.8)$ & $36.0(9.6)$ \\
Total cholesterol & $176(120-289)$ & $161(86-283)$ \\
LDL & $111(46.8-195)$ & $89(20-181)$ \\
In TNF (mean-centered) & $-0.32(-1.53$ to 3.24$)$ & $1.78(-1.90$ to 3.69$)$ \\
In IL-6 (mean-centered) & $-0.21(-2.67$ to 3.88$)$ & $1.62(0.29$ to 6.63$)$ \\
\hline
\end{tabular}

All variables are shown as means and standard deviations or proportions, except for TNF, IL-6, cholesterol, LDL, which are shown as median and $\mathrm{min} /$ max.

$B M I$ body mass index, PANSS positive and negative syndrome scale, $L D L$ low-density lipoprotein, In TNF natural log of tumor necrosis factor, In IL-6 natural log of interleukin-6. symptom severity in patients with schizophrenia as they could represent potential targets for novel therapeutics.

\section{METHODS}

Fifty-two chronic outpatients with a primary diagnosis of schizophrenia or schizoaffective disorder were recruited from the Atlanta Veterans Affairs Health Care System (Atlanta VAHCS) as part of two different parent studies. All subjects provided written informed consent for the study was approved by the Emory University Institutional Review Board and the Atlanta VAHCS Research and Development Committee. Inclusion/exclusion criteria as well as descriptions of the inflammatory markers have been previously described (see Supplementary Materials) ${ }^{17}$. Blood samples for inflammatory markers were obtained in chilled EDTA-coated tubes and spun at $2000 \times g$ for $15 \mathrm{~min}$ at $4^{\circ} \mathrm{C}$, and plasma was collected and stored at $-80^{\circ} \mathrm{C}$ for later batched analysis. Non-fasting lipid panels [total cholesterol, low-density lipoprotein (LDL), high-density lipoprotein (HDL), very low-density lipoprotein (VLDL), and triglycerides] were obtained from the electronic medical record for the date closest to the study visit (blood sampling and clinical interview). Negative symptoms, both total score and individual items were assessed using the Positive and Negative Syndrome Scale (PANSS).

Inflammatory markers were not normally distributed and were thus logtransformed. Moreover, as the inflammatory markers were run at different times, the concentrations were mean-centered (the batch effect was accounted for as a covariate in analyses). Median splits for both the metabolic and inflammatory markers were calculated in order to determine high and low groups. Linear regression models were used to determine the relationship between the interaction of metabolic and inflammatory markers with negative symptom severity including the following covariates: age, sex, race, smoking, body mass index (BMI), batch effect, and time between blood samples for metabolic and inflammatory markers. A replication sample including 58 individuals with schizophrenia was used to replicate the findings from the primary sample (see Supplementary Materials) ${ }^{14}$. a)

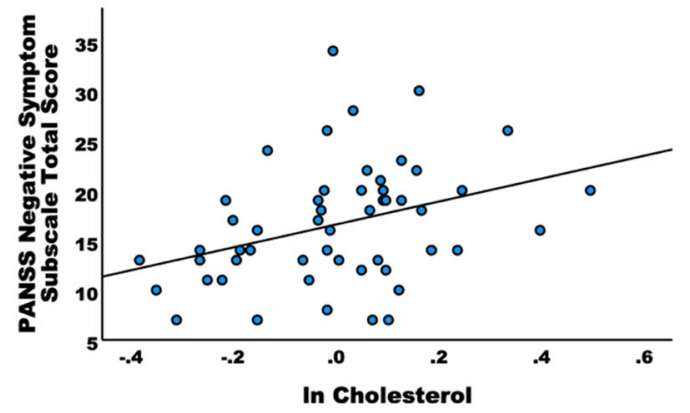

c)

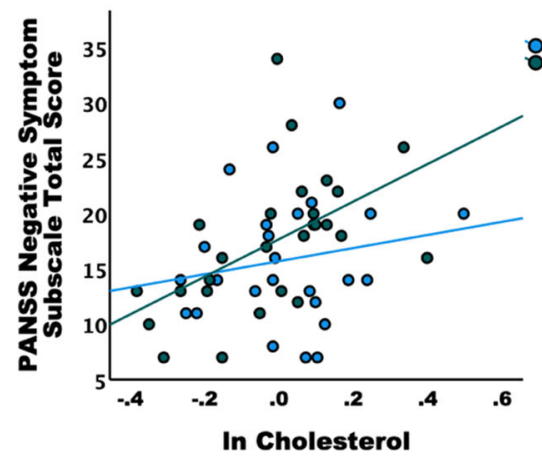

b)

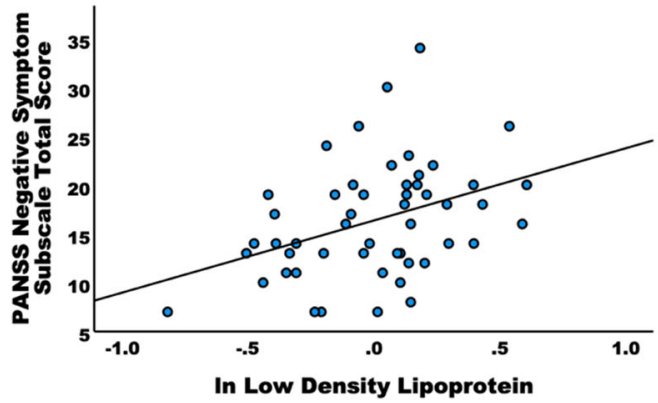

d)

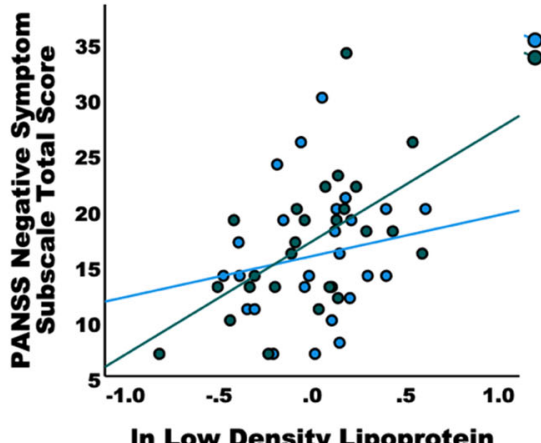

Fig. 1 Correlations between Positive and Negative Syndrome Scale (PANSS) Negative Symptom Subscale Score and total cholesterol and low-density lipoprotein (LDL). Of the metabolic markers, only (a) total cholesterol $(r=0.387, p=0.005)$ and $(\mathbf{b}) \mathrm{LDL}(r=0.356, p=0.009)$ were significantly correlated with the PANSS negative subscale score. The sample was split between those with high and low inflammation as indexed by a median split of a composite score of tumor necrosis factor (TNF) and interleukin-6 (IL-6). In the low inflammation subgroup (c), there was no relationship between PANSS Negative Symptom Subscale Score and cholesterol $(p=0.514)$ or LDL $(p=0.317)$, whereas in the high inflammation subgroup (d) significant correlations were found for total cholesterol $(p=0.001)$ and $\operatorname{LDL}(p=0.002)$. 


\section{Reporting Summary}

Further information on research design is available in the Nature Research Reporting Summary linked to this article.

\section{DATA AVAILABILITY}

The data that support the findings of this study are available from the corresponding author upon reasonable request.

Received: 4 June 2021; Accepted: 1 October 2021;

Published online: 20 October 2021

\section{REFERENCES}

1. Bekhbat, M. et al. Gene signatures in peripheral blood immune cells related to insulin resistance and low tyrosine metabolism define a sub-type of depression with high CRP and anhedonia. Brain Behav. Immun. 88, 161-165 (2020).

2. Goldsmith, D. R. et al. Protein and gene markers of metabolic dysfunction and inflammation together associate with functional connectivity in reward and motor circuits in depression. Brain Behav. Immun. 88, 193-202 (2020).

3. Nettis, M. A. et al. Metabolic-inflammatory status as predictor of clinical outcome at 1-year follow-up in patients with first episode psychosis. Psychoneuroendocrinology 99, 145-153 (2019).

4. Bekhbat, M. et al. Glucose and lipid-related biomarkers and the antidepressant response to infliximab in patients with treatment-resistant depression. Psychoneuroendocrinology 98, 222-229 (2018).

5. Pillinger, T. et al. Impaired glucose homeostasis in first-episode schizophrenia: a systematic review and meta-analysis. JAMA Psychiatry 74, 261-269 (2017).

6. Dickens, A. M. et al. Dysregulated lipid metabolism precedes onset of psychosis. Biol. Psychiatry 89, 288-297 (2021).

7. Goldsmith, D. R., Rapaport, M. H. \& Miller, B. J. A meta-analysis of blood cytokine network alterations in psychiatric patients: comparisons between schizophrenia, bipolar disorder and depression. Mol. Psychiatry https://doi.org/10.1038/mp.2016.3 (2016).

8. Goldsmith, D. R. \& Rapaport, M. H. Inflammation and negative symptoms of schizophrenia: implications for reward processing and motivational deficits. Front. Psychiatry 11, 46 (2020).

9. Felger, J. C. \& Treadway, M. T. Inflammation effects on motivation and motor activity: role of dopamine. Neuropsychopharmacol. 42, 216-241 (2017).

10. Page, K. A. et al. Effects of fructose vs glucose on regional cerebral blood flow in brain regions involved with appetite and reward pathways. JAMA 309, 63-70 (2013).

11. Mehta, D. et al. Transcriptional signatures related to glucose and lipid metabolism predict treatment response to the tumor necrosis factor antagonist infliximab in patients with treatment-resistant depression. Brain Behav. Immun. 31, 205-215 (2013).

12. Juckel, G. et al. Dysfunction of ventral striatal reward prediction in schizophrenia. Neurolmage 29, 409-416 (2006).

13. Goldsmith, D. R. et al. Association of baseline inflammatory markers and the development of negative symptoms in individuals at clinical high risk for psychosis. Brain Behav. Immun. 76, 268-274 (2019).

14. Goldsmith, D. R. et al. TNF-a and IL-6 are associated with the deficit syndrome and negative symptoms in patients with chronic schizophrenia. Schizophrenia Res. 199, 281-284 (2018).

15. Park, H. S., Park, J. Y. \& Yu, R. Relationship of obesity and visceral adiposity with serum concentrations of CRP, TNF-alpha and IL-6. Diabetes Res. Clin. Pr. 69, 29-35 (2005).

16. Popa, C., Netea, M. G., van Riel, P. L., van der Meer, J. W. \& Stalenhoef, A. F. The role of TNF-alpha in chronic inflammatory conditions, intermediary metabolism, and cardiovascular risk. J. Lipid Res. 48, 751-762 (2007).
17. Goldsmith, D. R. et al. Inflammatory markers are associated with psychomotor slowing in patients with schizophrenia compared to healthy controls. NPJ Schizophr. 6, 8 (2020).

\section{ACKNOWLEDGEMENTS}

Supported by grants from the Department of Veterans Affairs to E.D. ( 1101 CX000974-01A1, 1 I21 RX001897-01, and Merit Review Grant, Sensorimotor Gating in Schizophrenia) and from the National Institute of Mental Health to D.R.G. (K23 $\mathrm{MH114037).} \mathrm{Additional} \mathrm{grant} \mathrm{support} \mathrm{was} \mathrm{provided} \mathrm{to} \mathrm{E.D.} \mathrm{from} \mathrm{the} \mathrm{National} \mathrm{Institute}$ of Mental Health (1 R01 MH117315-01A1, R21 MH117512-01). Infrastructure support was provided by the Office of Research and Development, the Mental Health Service Lines, and the Center of Visual and Neurocognitive Rehabilitation at the Atlanta Veterans Affairs Medical Center, Decatur, GA. Additional infrastructure support was provided by the Department of Psychiatry and Behavioral Sciences of the Emory University School of Medicine, Atlanta, GA.

\section{AUTHOR CONTRIBUTIONS}

All authors made substantial contributions to the conception or design of the work or the acquisition, analysis, or interpretation of the data. All authors were involved in the drafting or revising of the manuscript and provided final approval of the completed version. All authors share accountability for the work as it relates to accuracy and integrity.

\section{COMPETING INTERESTS}

E.D. has received research support for work unrelated to this project from Auspex Pharmaceuticals, Inc. and Teva Pharmaceuticals, Inc. E.D. is a full-time attending psychiatrist and Nicholas Massa is a full-time research associate at the Atlanta Veterans Affairs Health Care System, Decatur, GA. The content is solely the responsibility of the authors and does not necessarily represent the official views of the Department of Veterans Affairs.

\section{ADDITIONAL INFORMATION}

Supplementary information The online version contains supplementary material available at https://doi.org/10.1038/s41537-021-00179-8.

Correspondence and requests for materials should be addressed to David $R$. Goldsmith.

Reprints and permission information is available at http://www.nature.com/ reprints

Publisher's note Springer Nature remains neutral with regard to jurisdictional claims in published maps and institutional affiliations.

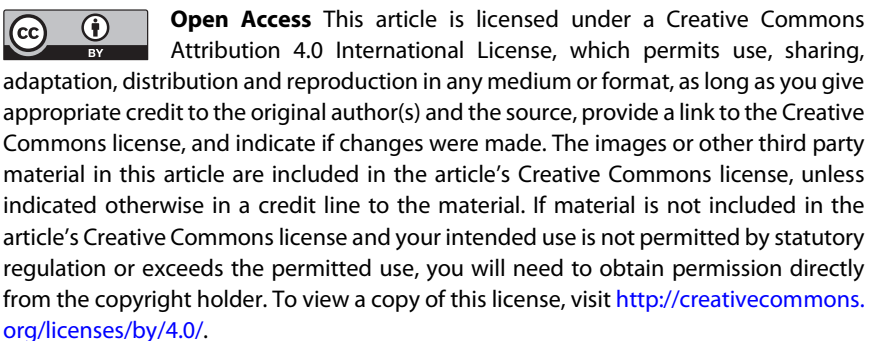

C) The Author(s) 2021 